\title{
Belt conveyor failure simulation at the design stage with transverse displacements of the belt
}

\author{
Vitalii Monastyrskyi ${ }^{1, *}$, Serhii Monastyrskyi ${ }^{1}$, Denis Nomerovskyi ${ }^{1}$, and Borys Mostovyi ${ }^{1}$ \\ ${ }^{1}$ Institute of Geotechnical Mechanics named by N. Poljakov of National Academy of Sciences of \\ Ukraine, 49005, Dnipro, Simferopolska Str., 2a, Ukraine
}

\begin{abstract}
To find possible conveyor failures at the design stage means to determine a transverse belt displacement and compare the obtained data with the permissible ones. The dynamic problem of the belt movement on the conveyor has been defined. Resistance and external forces, limits of the belt displacement have been determined. The transverse belt displacement can be described by partial differential equations. To solve the problem, the Fourier transform has been used. Change patterns in the transverse belt conveyor displacement dependent on conveyor's parameters, type of load, and skewing of the idlers along the conveyor have been obtained. The results agree with experimental data. The method of adaptive control of the transverse belt displacement has been described. The essence of this method is to adapt the model of the moving belt in the conveying trough to changed conditions and to reveal the uncertainty of the control with the known parameters of the mathematical model.
\end{abstract}

\section{Introduction}

Having analyzed the conveyors, which are used for transporting of bulk load, it has been revealed that failures caused by a transverse belt displacement, occur on conveyors with impact and suspended idlers. To improve the reliability of belt conveyors it is obligatory to clarify and eliminate the causes of the transverse belt displacement. The task becomes more complicated as the belt is characterized by very small static and dynamic stability. When the belt interacts with other elements of the conveyor by friction forces and is slightly disturbed it can lose its equilibrium state. It has been established [1-3] that the factors that cause the transverse belt displacement change with time and depend on the operating conditions of the equipment, its installation, the quality of the belts used, the design of loading devices, the distribution of the load, and climatic conditions. Most of these factors cause conveyor failures because of the occurrence of a transverse force, the value of which substantially depends on the adhesion of the belt to the idlers and the skewing angle in the plane of a belt movement. At mining enterprises failures occur due to the belt displacement in the transverse direction beyond the permissible limits, spills of material along the entire conveyor, additional wear of the belt, increased running resistance and lowered productivity of the belt conveyor performance. Therefore, designing conveyor belts, a specialist should pay a lot of attention to a reasonable choice

\footnotetext{
*Corresponding author: vfmonas@gmail.com
} 
of main dynamic system components, determining possible failures and revealing the uncertainty of external influence to stabilize transverse displacement under known parameters of the mathematical model.

The aim of the paper is to develop mathematical models to simulate possible conveyor failures (due to a transverse belt displacement) at the design stage and to develop a method for stabilizing a belt movement.

\section{Research methodology}

To discover potential causes of conveyor failures due to transverse belt displacement, the influence of various factors on the reliability of conveyor components and mechanisms as well as the selection of more significant factors with the help of correlation analysis and expert evaluation have been studied [1].

The above mentioned factors include: skewing of idlers, the material of the belt and idlers, external load, camber and flexural stiffness of the belt, suppleness of the idlers. Creation of the formula for a conveyor belt movement requires an idealized design model of a dynamic system that takes into account the influence of the significant factors. To represent the object of the research and dynamic characteristics of the system, the design model has been formalized. A number of assumptions have been suggested. These assumptions allow an adequate approximation of the research object to known design models, such as low-stiffness beam or a flexible thread. Belt camber is not usually taken into account. Belt stiffness is accounted for only in the cases with a big amount of padding. The influence of big cargo pieces over $0.2 B_{b}$ (where $B_{b}$ is the belt's width in meters) is not calculated unless they exceed $15 \%$ of the total volume of the cargo; the suppleness of the idlers is accounted for only in cases with amortizing or hinged idlers.

For modeling of potential conveyor failures due to a transverse belt displacement, a dynamic task of the belt movement has been formulated, resistance and external forces has been set, an acceptable transversal belt displacement has been defined. In this case, we have chosen a model without flexural stiffness, with an evenly distributed mass and load along the length of the conveyor, tension $S(\mathrm{~N})$, moving at the speed $v_{b}(\mathrm{~m} / \mathrm{s})$ along the conveyor that imitates idlers skewed according to the random law of distribution. Elasticity of the foundation underneath the belt is calculated with the coefficient $\varphi=1+2 S /\left(C_{r} l_{r}\right)$, where $C_{r}$ - the suppleness of the idlers $(\mathrm{N} / \mathrm{m}) ; l_{p}-$ the interval between idlers $(\mathrm{m})$. Resistance to a transverse belt movement on the track is assumed to be equal to the resistance to belt transverse movement on the idlers, and the movement on the trough is assumed to be unrestricted, i.e. the belt may move beyond the trough. External and restorative forces can transversally affect the belt. Transversal displacements on the drums are absent. Any changes to the time of the input parameters of the conveyor and the characteristics of the cargo load cause belt displacement along the longitudinal axis. The solution to the set problem is in finding the correlation between input and output parameters, under which transverse belt displacement parameter can excess an acceptable norm and a potential conveyor failure can be recorded.

Transversal forces acting on the belt are investigated in [4], where load intensity $p(x, t)$ $(\mathrm{N} / \mathrm{m})$ is represented as:

$$
p(x, t)=\left[\frac{a n l_{r}\left(q_{b}+q_{b c}\right)+F_{d i s t}(t)}{L}\right] \alpha(x),
$$

where $F_{\text {dist }}(t)$ - load on the idlers, changing over time, N; $a=10 \ldots .20$ - the proportionality coefficient; $n$ - the number of idlers in the conveyor system; pc.; $q_{b}, q_{b c}$ - linear loading of the belt and the cargo accordingly N/m; $L$ - length of the conveyor $(\mathrm{m}) ; a(x)=\alpha_{0} \sin (k \pi x / L)-$ 
the distribution law of skewed idlers alongside the conveyor; $\alpha_{0}=0.05 ; k=1,2, \ldots, n_{1}$.

With the size of the load taken into account, the formula for $F_{\text {dist }}(t)$ may be presented as the following [4]:

$$
F_{d i s t}(t)=\left[F_{0}+\sum_{i=1}^{\infty} \frac{\tau_{0}\left(m_{x}+\sqrt{D}\right)}{T_{0} \varphi} \frac{\sin \frac{i \pi \tau_{0}}{2 T_{0}}}{\frac{i \pi \tau_{0}}{2 T_{0}}} \cos \left(\omega_{i} t\right)\right]
$$

where $F_{0}-$ load affected by small cargo pieces, $\mathrm{N} ; m_{x}=\left(q_{b}+q_{b c}\right) l_{r}$ - mathematical expectation of the load affected by fines and small pieces $\left(q_{b}\right)$ and belt $\left(q_{b c}\right), \mathrm{N} ; D-$ load dispersion, $\mathrm{N}^{2} ; \varphi=1+2 S /\left(C_{r} l_{r}\right)$ - coefficient taking into account the stiffness of idlers $\left(C_{r}\right)$ and belt $\left(2 S / l_{r}\right) ; S$ - belt tension, $\mathrm{N} ; l_{r}$ - distance between idlers, $\mathrm{m} ; \tau_{0}, T_{0}$ - accordingly, the time of interaction of large lumps and idlers and the period of occurrence of this interaction in one of the idlers $\mathrm{s} ; \omega_{i}=k \pi i / T_{0}-$ the frequency of occurrence of an impulse of interaction with one idler, $1 / \mathrm{s} ; t$ - current time, $\mathrm{s} ; i$ - harmonics of interaction of large lumps with idler.

The resistance to the movement of the belt according to [4] is calculated with the help of the following:

$$
N_{b}=\frac{g}{4} b u \sin 2 \beta\left[\operatorname{tg} \beta+\operatorname{tg} \rho_{r e p}\right] L \gamma,
$$

where $N_{b}$ - resistance to a conveyor belt movement, $\mathrm{N} ; g$ - acceleration of gravity, $\mathrm{m} / \mathrm{s}^{2} ; b-$ idler length, $\mathrm{m} ; \beta$ - an angle of side idlers, deg; $\rho_{\text {rep }}$ - angle of the slope, deg; $u$ - the value of a belt displacement, $\mathrm{m} ; \gamma-$ load density, $\mathrm{kg} / \mathrm{m}^{3}$.

The mathematical description of the dynamic model of the process of a transverse belt displacement is formulated as the following: the belt in the form of a thread with a uniformly distributed load along the entire length moves in the longitudinal direction at a speed of $v_{b}$. The shape of the thread is determined by the guiding trough, which, being continuous, imitates the idlers, skewed along the conveyor length according to the law $\alpha(x)$. The resistance to the transverse movement of the thread along the trough is equal to the resistance to the transverse movement of the belt along the idlers. The thread movement in the trough is considered to be free. It is also supposed that the movement has the ability to go beyond the trough. The thread is open to the impact of external and restoring forces in the transverse direction. There is no transverse displacement of the thread on the drums. Any change in time of input parameters and load flow characteristics cause the thread to shift relatively to the longitudinal conveyor axis. In general, the description of the mathematical model of the transverse thread displacement in the conveyor trough can take the following form:

$$
\frac{\rho}{g} \frac{\partial^{2} u(x, t)}{\partial t^{2}}-S \frac{\partial^{2} u(x, t)}{\partial x^{2}}=p(x, t),
$$

where $\rho$ - external load density, $\mathrm{N} / \mathrm{m} ; u(x, t)$ - transverse displacement, $\mathrm{m}$.

The permissible value of the belt displacement is that, when exceeded, a possible failure of the conveyor is observed. In [4], the estimation scheme for determining the permissible transverse belt displacement, belt formalized model, and the limiting values of the belt displacement have been stated. The problem has been formulated in the following way: if there is an absence of a belt displacement (with the width of $B_{b}$ ), a material in the form of a trihedral prism occupies an equilibrated, central position. On each side of the belt there is the same gap $\Delta(\mathrm{m})$ between belt's edge and the material boundary on the belt. 
Recommended measurements of the gaps are $0,1 B_{b}$ [4] or $50-75 \mathrm{~mm}$ [2].

When the belt is displaced by the value of $U$, the material moves in the same direction by the value of $U$ and all points of the side face of the prism at a constant speed rotate around its top by the angle $\delta \rho_{\text {rep. }}$. In this case, the material becomes unstable, since the side face of the prism has an inclination angle exceeding $\delta \rho_{\text {rep }}$, which contradicts the real conditions. Therefore, the material can take a stable position at which the angle of inclination of the side face of the prism can again be equal to $\rho_{\text {rep }}$.

Equating the areas of geometric figures [4] before and after the transverse belt displacement, we can get the equation:

$$
\begin{aligned}
& U_{1}^{2}\left[\frac{\sin ^{2}\left(\beta+\rho_{r e p}\right) \operatorname{tg} \delta \rho_{r e p}}{\sin 4 \rho_{r e p}\left[\operatorname{tg} 2 \rho_{r e p}-\operatorname{tg} \delta \rho_{r e p}\right]}-\frac{1}{4} \sin 2\left(\beta+\rho_{r e p}\right)+\frac{1}{2} \frac{\sin ^{2}\left(\beta+\rho_{r e p}\right)}{\operatorname{tg} 2 \rho_{r e p}}\right]+ \\
& +I_{p} U_{1} \sin \left(\beta+\rho_{r e p}\right)-\frac{1}{2} l_{p}^{2} \frac{\operatorname{tg}\left(\beta+\rho_{r e p}\right) \operatorname{tg} \delta \rho_{r e p}}{\operatorname{tg}\left(\beta+\rho_{r e p}\right)-\operatorname{tg} \delta \rho_{\text {rep }}}=0 .
\end{aligned}
$$

where $U_{1}-$ load shift on a conveyor, m;

$$
\operatorname{tg} \delta \rho_{\text {rep }}=\frac{u \sin \left(\beta+\rho_{r e p}\right)}{l_{r}+u \cos \left(\beta+\rho_{r e p}\right)} .
$$

Solving the equation (5) regarding $U_{1}$, we can get the dependence of $U_{1}$ on the angle of the side idlers at fixed values of the belt displacement. Meanwhile, for side idlers angles $\left(10-40^{\circ}\right)$, the shift of the load might be accepted with sufficient accuracy:

$$
U_{1} \cong \frac{1}{2} U
$$

where $U$ - value of conveyor belt displacement, $\mathrm{m}$.

In the final form, the condition for the permissible transverse belt displacement can be:

$$
U \leq 2 \Delta
$$

The accuracy of mathematical models can be confirmed by the implementation of methods of mechanics and the theory of elasticity, as well as experimental verification of the main theoretical issues on the test bench and operating installations [1, 4]. It has been found that the theoretically obtained dependences are in good agreement with experimental data, when transported cargo is in size up to $0.1 B_{b}$.

\section{Results and discussion}

In order to model possible conveyor failures due to transverse belt displacement under the influence of various factors, the dynamic model (4), external influence (1), resistance forces (3) and permissive values of transverse belt displacement (7) have been chosen. The skewing function of the idlers has been taken as $\alpha(x)=\sin (2 \pi x L)$. The solution of the nonhomogeneous equation (4) has the form:

$$
u(x, t)=\sum_{k=0}^{\infty} \sin \left(\frac{k \pi}{L} x\right)\left[A_{0 k}+A_{1 k}+\sum_{v=1}^{\infty} A_{z v k} \cos \left(\omega t-\alpha_{v}\right)\right],
$$


where $\alpha_{v}-$ non-dimensional quantity derived from the initial conditions. The coefficients $A_{0 k}, A_{1 k}$ and $A_{z v k}$ have been given in [4].

Possible failures of transverse belt displacement under bulk influence have been determined by comparing the solutions of the second-order partial differential equations with the right-hand side of (4) using the Fourier transform under the following initial and boundary conditions:

$$
\text { if } t=0 \quad u=0 ; \quad \frac{\partial u}{\partial t}=0 ;\left.u\right|_{x=0}=0 ;\left.u\right|_{x=L}=0 .
$$

The following initial data have been taken: $a=20 ; q_{b c}=2000 \mathrm{~N} / \mathrm{m} ; L=100 \mathrm{~m}$ and $50 \mathrm{~m}$; $\rho=200 \mathrm{~N} / \mathrm{m} ; \quad S=53000 \mathrm{~N} ; \quad T_{1}=0.3 \mathrm{~s} ; \quad v_{b}=2 \mathrm{~m} / \mathrm{s} ; \quad \gamma=3200 \mathrm{~kg} / \mathrm{m}^{3} ; \quad \beta=0.698 \mathrm{rad} ;$ $\rho_{\text {rep }}=0.5236 \mathrm{rad} ; m_{x}=700 \mathrm{~N} ; n=100$ and 50 pcs.; $\sqrt{D}=150 \mathrm{~N} ; \tau_{0}=1.5 \mathrm{~s} ; T_{0}=1.0 \mathrm{~s}$; $\omega=2 \pi / T_{1}$.

The results of the transverse belt displacement, depended on the load, are presented in Figure 1.
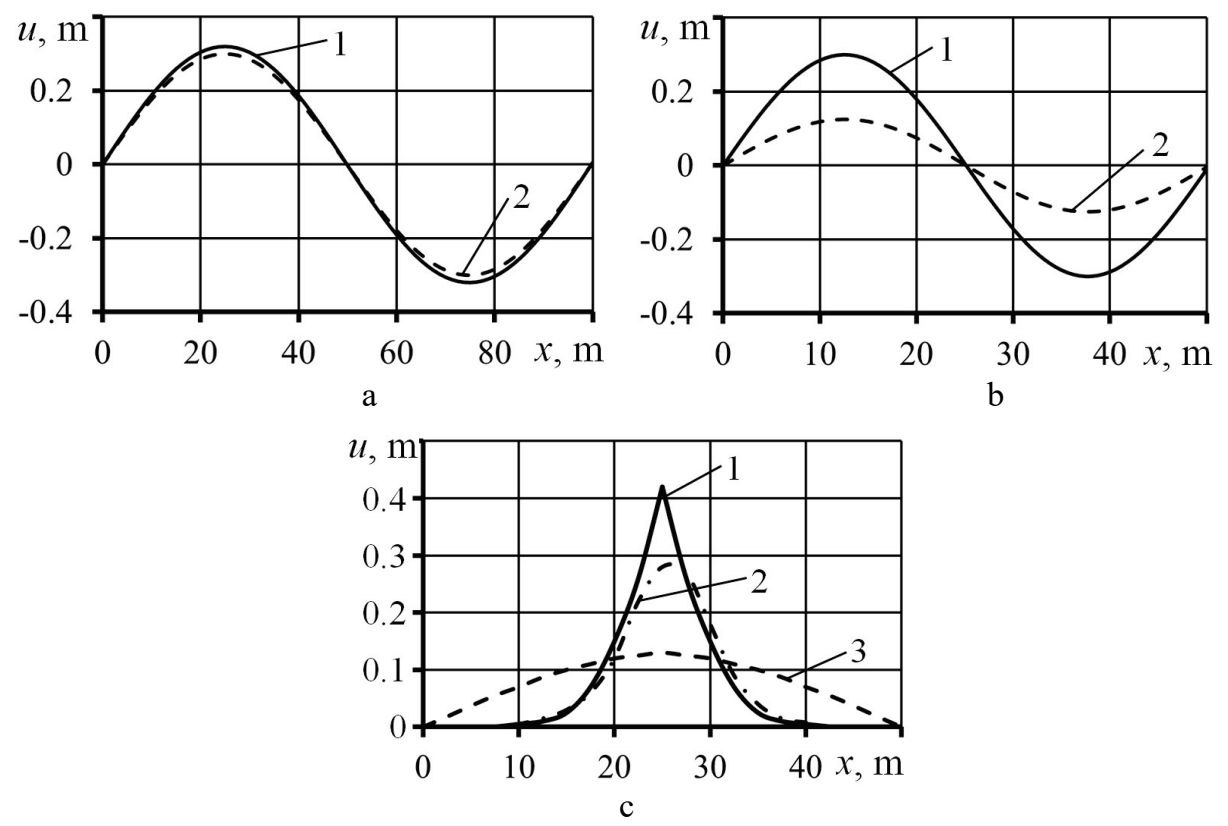

Fig. 1. The dependence of the transverse belt displacements on conveyor's length: $a, b$ - respectively on conveyors (100 and $50 \mathrm{~m}$ long) with a distributed load without taken into account $F_{\text {dist }}(t)$ (curve 1 ) and with taken into account $F_{\text {dist }}(t)$ (curve 2); c - with a local load on a belt (50 m long) with taken into account $F_{\text {dist }}(t)$ (curve 1 ) and without taken into account $F_{\text {dist }}(t)$ (curve 2); curve 3 - experimental.

Modeling of transverse belt displacements on the conveyors of different length, with distributed and local load, revealed that:

- the form of the transverse belt displacement significantly depends on the distribution skewed idlers along conveyor's length and the type of load (Fig. 1), and practically does not depend on time;

- possible conveyor failures with transverse belt displacement occur when the belt width is $B_{b} \leq 1400 \mathrm{~mm}$, since the maximum displacement of $300 \mathrm{~mm}$ exceeds the permissible $0.2 B_{b}$ (Fig. 1). Therefore, when designing a conveyor for given conditions, it is necessary to choose a belt width of at least $1600 \mathrm{~mm}$; 
- a local load on the conveyor belt (Fig. 1, c) causes a transverse belt displacement 1.3 times greater than uniformly distributed load does (Fig. 1, a, b), as a result it increases the risk of a possible conveyor failure with the same width of the belt.

The experimental research methodology is given in [4]. Skewed idlers were installed on the conveyor according to the $\alpha_{0} \sin (k \pi x / L)$ law, the $x$-distributed load, and the local load was created with the help of one skewed idler at $x=L / 2$. The calculated curves were obtained taking into account the parameters of the conveyors of 100 and $50 \mathrm{~m}$ long.

\section{Control of the process of transverse belt displacements}

On the basis of the studies of a belt movement in the conveyor trough, we have developed a method aimed at maintaining the stable movement of a belt along the idlers with the known parameters of the dynamic model, when the following condition is met:

$$
u(x, t) \leq[u],
$$

where $u(x, t),[u]$ - belt displacement and its permissible value correspondingly, $\mathrm{m}$.

If the belt displacement does not go beyond the condition (10), then a belt movement is considered stable, since dissipative forces do not allow the belt to go beyond the zone of equilibrium position. Yet any change in time of the input conveyor parameters or the characteristics of the load flow causes the belt to move towards the longitudinal axis. Variable input parameters include linear load on the idlers, belt tension, skew of the idlers along the conveyor belt, and the value of the eccentric load. To control the stability of the belt movement it is necessary to adapt a dynamic model to changed operating conditions and disclose the uncertainty of the control action with known parameters of the mathematical model. In this case, the condition of stable belt movement in the trough must be followed (10).

In [6], a method of adaptive control of transverse belt displacement was described, for the implementation of which a dynamic model was used (4).

To solve the issue, it is obligatory to find the correlation between the input and output parameters, where the transverse belt displacement can be within a certain zone of a belt's stable movement in the trough. Solving the equation (4), we have taken into account the boundary conditions and that there is no acceleration of the transverse belt displacement $u=[u]$ :

$$
\left.u\right|_{x=0}=0 ;\left.\quad u\right|_{x=L}=0
$$

can get:

$$
S=\frac{a n l_{r} \alpha_{0}}{[u] k \pi}\left(q_{b}+q_{b c}\right) \cdot\left(x-\frac{L}{k \pi} \sin \frac{k \pi}{L} x\right),
$$

where $k=1,2,3, \ldots, n-$ is the harmonic index of the skewed idlers distribution.

As it can be derived from (12), if the permissible belt displacements and fixed values of the loads on the idlers are provided, the tension of the belt is to change within the limits at which the restoring force exceeds the forces that cause the belt to get side-tracked, and a steady movement of the belt is observed within the limits of permissible displacements.

In cases where the internal restoring forces are insufficient to return the belt to the area of its stable movement, an external control action is applied, in which equation (4) takes the form: 


$$
S \frac{d^{2} u(x)}{d x^{2}}+\frac{a n l_{r} \alpha_{0}}{L}\left(q_{b}+q_{b c}\right) \cdot \sin \frac{k \pi}{L} \cdot x=-p(u),
$$

where $p(u)$ - external control applied to the belt [4], which is an approximation of the linear dependence of the experimental data presented in Figure 2 (curves 1, 2, 3, 4), N/m.

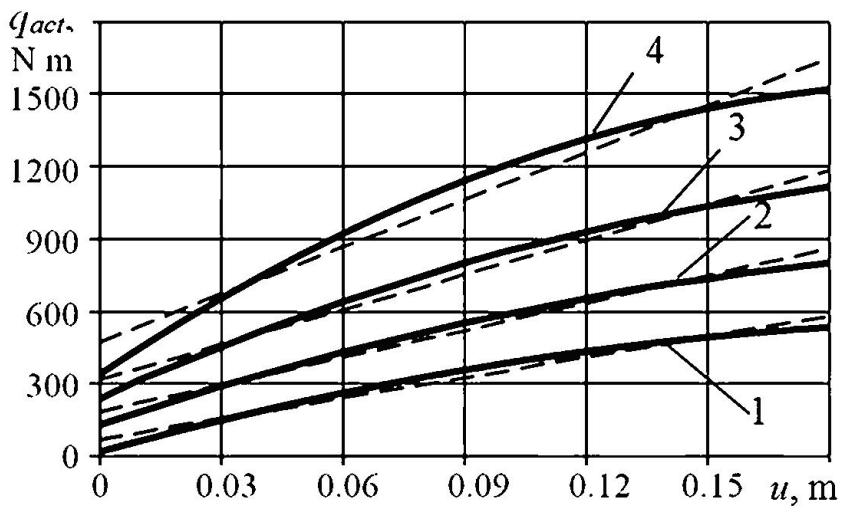

Fig. 2. Experimental dependences of the impact $\left(q_{a c t}\right)$ on the belt of skewed idlers on the parameters of the conveyor and characteristics of the load flow (solid lines): $1-S=25000 \mathrm{~N}, q_{b c}=1000 \mathrm{~N} / \mathrm{m}$; $2-S=30000 \mathrm{~N}, q_{b c}=1500 \mathrm{~N} / \mathrm{m} ; 3-S=60000 \mathrm{~N}, q_{b c}=2000 \mathrm{~N} / \mathrm{m} ; 4-\mathrm{S}=80000 \mathrm{~N}, q_{b c}=3000 \mathrm{~N} / \mathrm{m}$. The dashed line shows the corresponding approximating curves.

The experimental research technique has been given in [4]. The impact on the belt was made by a skewed idler, the belt displacement was measured with the help of a displacement sensor, the force was recorded by a deflector idler with a strain gauge.

The regression equations for various parameters of the conveyor and the characteristics of the load flow, approximating curves 1,2, 3, 4 in Figure 2 with a dependence of the type $p(u)=A \cdot u+B$, are presented as the following:

$$
\begin{aligned}
& p_{1}(u)=2840 u+72.78 \\
& p_{2}(u)=3750 u+185.5 \\
& p_{3}(u)=4800 u+320.2 \\
& p_{4}(u)=6520 u+476.2 .
\end{aligned}
$$

The solution of the nonhomogeneous differential equation (13) with the boundary conditions (10) is described in detail in [6] and is presented in general form for $x=L / 2$ :

$$
u(x=L / 2)=\frac{B}{A}\left[\frac{2 \operatorname{sh}\left(\frac{L}{2} \sqrt{\frac{A}{S}}\right)}{\operatorname{sh}\left(L \sqrt{\frac{A}{S}}\right)}-1\right]-\frac{p(x)}{S\left(\frac{k^{2} \pi^{2}}{L^{2}}\right)+A} \sin \left(\frac{k \pi}{2}\right),
$$

where $A\left(\mathrm{H} / \mathrm{m}^{2}\right)$ and $B(\mathrm{H} / \mathrm{m})$ - approximation coefficients in $(14)-(17) ; \sqrt{A / S}-$ roots of the characteristic equation [6]; $p(x)=\left[\operatorname{anl}_{r}\left(q_{b}+q_{b c}\right) / L\right] \alpha(x)$ - distributed load with skewed idlers taken into account, $\mathrm{N} / \mathrm{m}$.

Modeling the process, the following initial data were taken: $a=20 ; q_{b c}=2000 \mathrm{~N} / \mathrm{m}$; $l_{r}=1.0 \mathrm{~m} ; n=100 ; L=100 \mathrm{~m} ; \rho=300 \mathrm{~N} / \mathrm{m} ; q_{b}=200 \mathrm{~N} / \mathrm{m} ; S=53000 \mathrm{~N} ; \alpha_{0}=0.05$. 
Using the equation (18) and changing the values of the approximation coefficients $A$ and $B$ when the condition $u(x) \leq[u]$ is fulfilled, we obtain the regime of the stable belt movement in the conveyor trough (Fig. 3, curve 3).

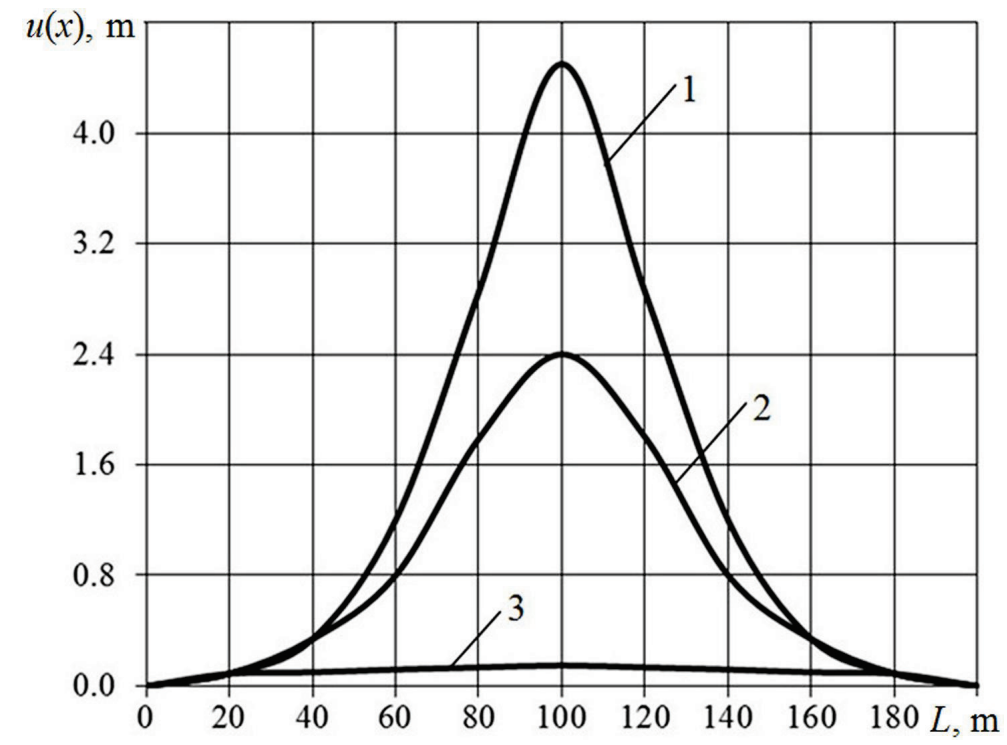

Fig. 3. Dependences of changes of transverse belt displacements along the length of the conveyor: $1-p_{1}(u) ; 2-p_{2}(u) ; 3-p_{3}(u)$.

The larger the approximation coefficients $A$ and $B(18)$ are, the more effective the control action is. To stabilize the belt movement of long conveyors $(L>500 \mathrm{~m})$, it is necessary to take into account the range of the control action, in which stabilization of the belt movement within the limits of $u(x) \leq[u]$ is achieved along the entire conveyor. If this condition is not met along the conveyor at any point, then a second, third, fourth and so on control action is assigned. The interval between the points depends on the belt tension, the characteristics of the belt and load flow, as well as on the effectiveness of the impact. In real conditions, the control action can be created using a centering idler equipped either with or without a drive.

\section{Conclusions}

1. Possible conveyor failures due to transverse belt displacements occur if the displacements exceed the permissible values. In this case, the form of the change in the position of the conveyor belt depends on the skew of the idler along its length, the parameters of the conveyor and the characteristics of the load flow, the type of load, and has a slight impact of time.

2. A model which helps to simulate possible conveyor failures at the design stage with transverse belt displacements has been developed. The essence of the model is to find the correlation between input and output parameters, in which the transverse belt displacements can go beyond the limits and a possible conveyor failure can be recorded.

3. A method to control the transverse belt displacements has been developed. The essence of the method is to adapt the dynamic model of the moving belt in the conveyor trough to changing conditions and to disclose the uncertainties of the control action with the known parameters of the mathematical model. 


\section{References}

1. Monastyrsky, V.F. (1990). Development of methods and tools for managing the reliability of high-power belt conveyors. D. Sc. Thesis, Mining Machines, Institute of Geotechnical Mechanics named by N. Poljakov of National Academy of Sciences of Ukraine, Dnepropetrovsk, Ukraine

2. Galkin, V. I., Dmitriev, V. G., Dyachenko, V. P. (2005). Sovremennaya teoriya lentochnykh konveyerov gornykh predpriyatiy. Moskva: MGGU

3. Yakhontov, Iu. F. (2005). The development of the theory and methods for calculating belt conveyors with suspended idlers for mining enterprises. D. Sc. Thesis, Mining Machines, Moskva, Russia

4. Monastyrsky, V. F. (1975). Investigation of ways to reduce transversal displacements of the belt on a loaded branch of conveyors, transporting large-sized loads at mining enterprises. Abstract of Ph. D. dissertation, Mining Machines, Institute of Geotechnical Mechanics of National Academy of Sciences of Ukraine, Dnepropetrovsk, Ukraine

5. Monastyrsky, V. F., Kiriya, R.V., Smirnov, A. N. (2017). Vybor znacheniy koefitsienta dinamichnosti vzaimodeystviya gruza s rolikooporami konveyera. Geo-Technical Mechanics, 137, 191-199

6. Monastyrsky, V. F., Kiriya, R. V., Maksiutenko, V. Yu., Smirnov, A. N. (2011). Adaptive of the belt transversal displacement control on conveyor. Proc. of the International scientific conference "Forum of Mining Engineers", National Mining University, Dnepropetrovsk, October, 234-240 